\title{
Estado de Mistura e Dispersão da Fase Borrachosa em Blendas PVC/NBR
}

\author{
Fábio R. Passador, Luiz A. Pessan \\ Departamento de Engenharia de Materiais, UFSCar

\begin{abstract}
Antonio Rodolfo Jr. Braskem S/A
\end{abstract}

Resumo: Termoplásticos modificados com elastômeros têm despertado grande interesse de pesquisadores e de indústrias devido à atraente relação custo/benefício e a possibilidade de aumento significativo da tenacidade sob impacto de polímeros frágeis com a incorporação de uma fase borrachosa dispersa. Este artigo mostra a relação entre o estado de mistura e a dispersão da fase borrachosa em blendas de poli (cloreto de vinila) (PVC) com borracha nitrílica (NBR). O estado de mistura de blendas poliméricas é um fator muito importante a ser considerado no desenvolvimento destes tipos de materiais, uma vez que determina o nível de mistura molecular das blendas. A característica estrutural do PVC é responsável pela morfologia das blendas PVC/NBR, sendo que há uma otimização de propriedades mecânicas quando as partículas de borracha formam a morfologia "pseudo-network", ou seja, quando as partículas de borracha estão randomicamente dispersas entre as partículas primárias do PVC.

Palavras-chave: Poli (cloreto de vinila), PVC, borracha nitrílica, NBR, blenda PVC/NBR, estado de mistura, microrreologia, dispersão de fase borrachosa.

\section{Phase Separation and Rubber Phase Dispersion in PVC/NBR Blends}

Abstract: Thermoplastics modified with elastomers have attracted great interest of researchers and industries due to the low cost/benefit relationship and the possibility of significant increase in the toughness of brittle polymers through the incorporation of a dispersed rubber phase. This article shows the relationship between the interaction between phases and rubber phase dispersion in poly (vinyl chloride)/nitrile rubber blends.

The interaction between components in polymer blends is an important factor to be considered in the development of this kind of materials because it determines the level of the mixture at the molecular level. The particulate nature of PVC and the degree of nitrile groups in the NBR are responsible to the PVC/NBR blends morphologies leading to an optimization of the mechanical properties when the rubber particles form a pseudo-network morphology with the rubber particles randomly dispersed in between the PVC primary particles.

Keywords: Poly (vinyl chloride), PVC, nitrile rubber, NBR, PVC/NBR blend, miscibility, phase interaction, microrheology, rubber phase dispersion.

\section{Introdução}

\section{Blendas Poliméricas}

Nas últimas décadas, a busca de novos materiais com propriedades específicas a cada aplicação e comercialmente viáveis tem despertado grande interesse de pesquisadores. As blendas poliméricas têm se mostrado uma excelente alternativa, uma vez que as propriedades físicas e químicas podem ser alteradas possibilitando a obtenção de materiais poliméricos para uma ampla gama de aplicações ${ }^{[1]}$.

Blendas poliméricas são sistemas poliméricos originários da mistura física de dois ou mais polímeros e/ou copolímeros, sem que haja um elevado grau de reações químicas entre eles. Para ser considerado uma blenda, os compostos devem ter concentração acima de $2 \%$ em massa do segundo componente ${ }^{[2]}$.

\section{Métodos de Obtenção de blendas poliméricas}

Uma classificação de blendas poliméricas pode ser feita através dos métodos de obtenção, havendo, dessa forma, três tipos de blendas: por solução, por reticulados poliméricos interpenetrantes (IPN) e por mistura mecânica no estado fundido ${ }^{[1,2]}$.

As blendas por solução são obtidas através da preparação de soluções individuais de cada polímero em um solvente comum, com posterior mistura das soluções nas

Autor para correspondência: Fábio R. Passador, Departamento de Engenharia de Materiais, UFSCar, Via Washington Luís, km 235, Caixa Postal 676, CEP:13565-905, São Carlos, SP. E-mail: fpassador@polvo.ufscar.br 
proporções desejadas. O aquecimento pode ser utilizado para aumentar o grau de solubilidade dos componentes individuais ou da mistura. A etapa mais importante é a evaporação do solvente, que normalmente é feita através da formação de um filme e posterior evaporação à temperatura ambiente, em estufa ou sob vácuo. Esse tipo de blenda é de baixa produtividade e geralmente são desenvolvidas em laboratório ${ }^{[1,2]}$.

As blendas por reticulado polimérico interpenetrantes (IPN ou interpenetratig networks) são obtidas por uma mistura polimérica onde os constituintes estão na forma de reticulados que se interpenetram e formam um único reticulado, sem que haja qualquer tipo de reação química entre eles. Quando somente um dos constituintes estão na forma reticulada este tipo de blendas são conhecidos como semi-IPNs, como exemplo a blenda PP/EPDM. Os INPs são tipos especiais de blendas poliméricas e são utilizados para melhorar a interação entre fases e a compatibilidade de blendas por solução e por mistura mecânica ${ }^{[1,2]}$.

As blendas por mistura mecânica no estado fundido ou melt blending são obtidas pela mistura dos componentes poliméricos em seu estado fundido ou amolecido. A mistura mecânica envolve aquecimento e alto cisalhamento; é o método mais utilizado industrialmente por razões econômicas e porque permite a mistura de polímeros em grande escala ${ }^{[1,2]}$.

\section{Estado de Mistura de blendas poliméricas}

Apenas a mistura entre os dois polímeros não garante a formação de uma blenda com as propriedades desejadas. Uma das características importantes a ser considerada em uma blenda polimérica é a miscibilidade ou grau de interação entre seus componentes, que corresponde ao nível de mistura molecular entre os polímeros ou fases constituintes do sistema ${ }^{[2,3]}$. A miscibilidade ou o grau de interação entre as possíveis fases presentes no sistema determinam direta ou indiretamente o comportamento do novo material desenvolvido.

As blendas são miscíveis quando os segmentos moleculares dos componentes poliméricos se misturam intimamente sem que haja qualquer segregação entre as moléculas, ou seja, são homogêneas em escala molecular. No caso de blendas imiscíveis há a formação de mais de uma fase, onde nenhuma molécula de uma fase se encontra intimamente ligada à fase vizinha ${ }^{[2,3]}$. Alguns aspectos básicos sobre miscibilidade em sistemas poliméricos são abordados a seguir para um melhor entendimento do tema deste trabalho sobre estado de mistura e dispersão da fase borrachosa em blendas $\mathrm{PVC} / \mathrm{NBR}$.

Termodinamicamente, o comportamento de equilíbrio de fases em misturas é controlado pela variação da energia livre de mistura, através da equação de energia livre de Gibbs:

$$
\Delta G_{m}=\Delta H_{m}-T \Delta S_{m}
$$

Onde $\Delta G_{m}$ é a variação molar de energia livre de mistura, $\Delta H_{m}$ é a variação molar de entalpia de mistura, $T$ é a temperatura absoluta e $\Delta S_{m}$ é a variação molar de entropia de mistura.

Analisando a equação de energia livre de mistura, é possível obter três situações diferentes para sistemas poliméricos: (a) se $\Delta G_{m}>0$, o sistema será imiscível, havendo duas ou mais fases presentes;

(b) se $\Delta G_{m}=0$, o sistema estará em equilíbrio dinâmico;

(c) se $\Delta G_{m}<0$, o sistema será miscível, constituído por uma única fase.

Para que o sistema seja miscível e estável, além de $\Delta G_{m}<0$, deve satisfazer a relação:

$$
\left(\frac{\partial^{2} \Delta G_{m}}{\partial \phi_{i}^{2}}\right)_{T, P}>0
$$

Onde $\phi_{i}$ é a fração volumétrica de cada componente $i$. Esta relação assegura a estabilidade contra a separação de fases.

A energia livre de mistura é dependente da temperatura e da composição. A Figura 1 apresenta esta dependência em três temperaturas.

Em $T_{1}$, as condições para miscibilidade de blendas poliméricas são satisfeitas, ocorrendo misturas miscíveis e monofásicas para todas as composições.

Em $T_{2}$, a segunda condição (equação 2) não é satisfeita para todas as composições, e misturas entre $B$ e $B$ ' separamse em duas fases, uma vez que a energia livre total é mais baixa que a da fase homogênea. $T_{c}$ é uma temperatura intermediária, correspondente ao ponto crítico $C$. Na Figura 1(a), $T_{1}>T_{2}$, e $T_{c}$ é a temperatura crítica máxima de solução (UCST); na Figura 1(b), $T_{2}>T_{I}$ e $T_{c}$ é uma temperatura crítica mínima de solução (LCST). O intervalo de todos os pontos entre $B$ e $B$ 'é uma curva binodal. Os pontos de inflexão $S$ e $S$ ' na curva de energia livre para $T_{2}$ definem uma curva espinodal. A curva binodal define o comportamento de equilíbrio de fases, enquanto que a curva espinodal está relacionado com mecanismo e cinética de processos de separação de fases ${ }^{[4]}$.

Quanto à formação da morfologia, o mecanismo binodal, em seus primeiros estágios, origina uma morfologia em que uma fase descontínua está dispersa em uma fase contínua, enquanto que no mecanismo espinodal a morfologia resultante é formada por duas fases contínuas simultaneamente ${ }^{[4]}$.

Para mistura de polímeros, $\Delta \mathrm{G}_{\mathrm{m}}$ depende essencialmente de $\Delta \mathrm{H}_{\mathrm{m}}$, que por sua vez depende da interação molecular e $\Delta \mathrm{S}_{\mathrm{m}}$ se anula para mistura de líquidos com elevada massa molar. Assim, quanto maior for a interação entre os polímeros mais $\Delta \mathrm{H}_{\mathrm{m}}$ se aproximará de zero, tornando $\Delta \mathrm{G}_{\mathrm{m}}$ sempre negativo, ou seja, a blenda será sempre miscível.

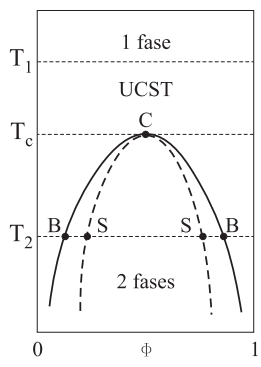

(a)

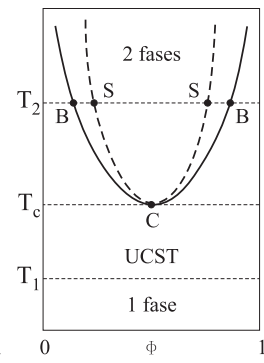

(b)

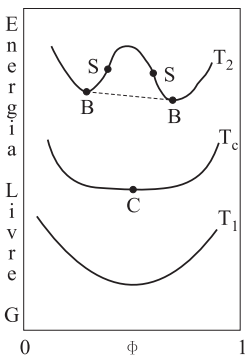

(c)
Figura 1. Diagrama de fases para misturas: (a) UCST; (b) LCST; (c) dependência da energia livre de mistura com a composição para as temperaturas acima e abaixo do valor crítico 
Blendas PVC/NBR possuem uma particularidade devido à presença da acrilonitrila na borracha nitrílica. A interação molecular da blenda PVC/NBR aumenta a medida que o conteúdo de AN aumenta na composição de NBR.

As blendas poliméricas imiscíveis podem apresentar diferentes tipos de morfologias de fases, entre elas, uma fase dispersa numa matriz contínua ou ambas as fases simultaneamente contínuas, conhecida como morfologia co-contínua. No caso da blenda PVC/NBR onde o teor de AN na borracha é insuficiente para tornar o sistema polimérico miscível é possível obter estes tipos de morfologia. Para melhorar a tenacidade do PVC a morfologia adequada é aquela onde a fase NBR torna-se dispersa na matriz contínua de PVC. A morfologia de blendas poliméricas é afetada por diversos fatores (tamanho, forma e teor da fase dispersa; condições de processamento; entre outros). Uma maneira de compreender a morfologia gerada é através da correlação desta com o comportamento reológico das blendas ${ }^{[1]}$.

\section{Microrreologia de blendas poliméricas}

A microrreologia é uma área da reologia que tem como objetivo correlacionar as propriedades reológicas macroscópicas, de componentes individuais e de um sistema disperso, como viscosidade e elasticidade, com uma descrição detalhada de mudanças em elementos de volume durante o fluxo. Dessa forma, é possível correlacionar as características reológicas da mistura com a morfologia final da blenda ${ }^{[2-4]}$.

Taylor ${ }^{[5]}$ estendeu as pesquisas de Einstein sobre a deformação e quebra da gota de líquidos em meios líquidos. Uma forma de compreender o comportamento sob fluxo de blendas poliméricas é através da dispersão de uma gota de um líquido Newtoniano em outro líquido Newtoniano, por exemplo, gotas de óleo em água, sujeitos a campos deformacionais bem definidos. A Figura 2 apresenta a deformação de uma gota segundo a teoria de Taylor.

Taylor observou que a baixas taxas de deformação em ambos os campos de fluxo há deformação da gota esférica. O comportamento das gotas é influenciado por dois fatores: razão de viscosidade e número capilar.

A razão de viscosidade $(\lambda)$ entre a gota dispersa e a matriz, que é dada por:

$$
\lambda=\frac{\eta_{d}}{\eta_{m}}
$$

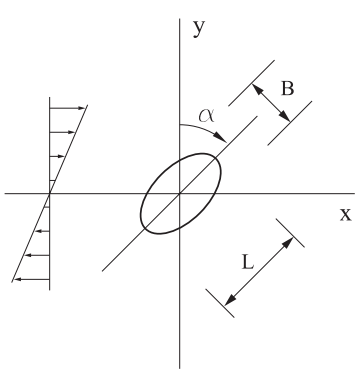

(a)

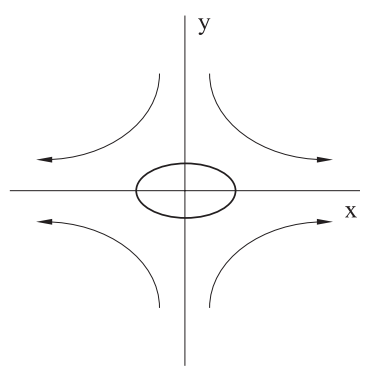

(b)
Figura 2. Esquema da deformação de gotas: (a) em fluxo cisalhante uniforme; (b) em campos de fluxo hiperbólicos planos ${ }^{[5]}$
Onde $\eta_{d}$ e $\eta_{m}$ são as viscosidades da fase dispersa e da matriz, respectivamente.

O número de capilar $(k)$, por sua vez, é dado por:

$$
k=\frac{\sigma d}{v_{12}}=\frac{\eta_{\mathrm{m}} \dot{\gamma} d}{v_{12}}
$$

Onde $\sigma$ é a tensão de cisalhamento local, $\dot{\gamma}$ é a taxa de cisalhamento, $d$ é o diâmetro da gota e $v_{12}$ é a tensão interfacial entre a fase dispersa e a matriz ${ }^{[5,6]}$.

Em condições de baixas taxas de cisalhamento a deformabilidade $(D)$ e o ângulo de orientação $(\alpha)$ da gota podem ser dados, respectivamente, pelas equações 5 e 6 :

$$
\begin{aligned}
& D=\frac{(L-B)}{(L+B)} \approx E=\frac{\sigma_{12} d}{v} \frac{(19 \lambda+16)}{(16 \lambda+16)} \\
& \alpha=\frac{\pi}{4}
\end{aligned}
$$

Onde $B$ e $L$ são a largura e o comprimento do esferóide apresentado na Figura 2, e $E$ é um número adimensional. Para pequenas deformações, a condição crítica para a quebra de uma gota aproximadamente esférica é $E \geq 1$.

Muitos trabalhos foram desenvolvidos correlacionando a razão de viscosidade com o fenômeno de quebra de gotas dispersas. Tomotik ${ }^{[5]}$ investigou um mecanismo para dispersar um líquido em outro através da teoria de crescimento de instabilidade capilar de Rayleigh em uma fibrila de um material Newtoniano, em um segundo fluído Newtoniano.

A Figura 3 apresenta a instabilidade senoidal da fibrila, onde são definidos o raio inicial $\left(R_{0}\right)$, o raio médio da fibrila $(\bar{R})$, a amplitude de distorção $(A)$ e o comprimento de onda da instabilidade senoidal $(\Lambda)$.

O raio médio da fibrila $(\bar{R})$ é dado por:

$$
\bar{R}=\left[\frac{\left(R_{0}^{2}-A^{2}\right)}{2}\right]^{\frac{1}{2}}
$$

A teoria de Rayleigh, por sua vez, é descrita por:

$$
R_{(z)}=\bar{R}+A \sin \left(\frac{2 \pi z}{\Lambda}\right)
$$

A Figura 3 mostra que, quando o comprimento de onda da instabilidade senoidal $(\Lambda)$ for maior que o perímetro da seção transversal da fibrila $\left(2 \pi R_{0}\right)$, as fibrilas tornam-se instáveis e a tensão interfacial diminui com o aumento da amplitude de distorção, não sendo possível manter-se a coesão das moléculas.

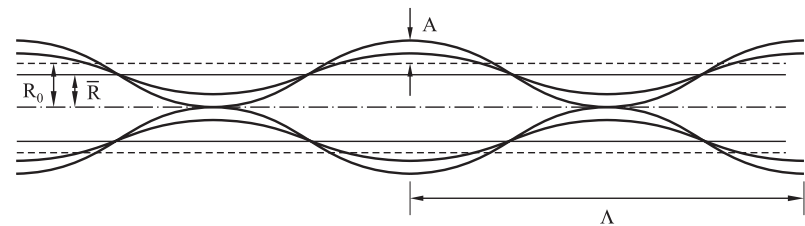

Figura 3. Instabilidade senoidal da fibrila ${ }^{[5]}$ 
Ocorre desta forma o rompimento da fibrila, gerando, conseqüentemente, uma série de partículas pequenas em linha. A amplitude de distorção $(A)$, nesse caso, deve crescer exponencialmente com o tempo, sendo que o rompimento das fibrilas ocorrerá se o tempo de mistura for maior que o tempo de ruptura e/ou a amplitude da distorção for maior que o raio médio da fibrila.

Através dos trabalhos de Taylor e Tomotika, pode-se concluir que o comportamento microrreológico de um sistema de líquidos Newtonianos bifásicos auxilia na compreensão do processo de deformação e quebra de gotas durante a mistura numa extrusora.

Nos estágios iniciais de mistura no processamento por extrusão ocorre a fusão dos componentes poliméricos macroscópicos, tornando uma mistura de líquidos, enquanto a deformação viscosa é imposta na mistura fundida pela rotação da rosca de extrusão ${ }^{[7]}$. Durante a fusão dentro da extrusora, as partículas de polímeros constituem um leito sólido pela interface sólido/fundido e emerge no modelo de fluxo fundido. As camadas de fundido são submetidas às deformações geradas pelo fluxo de arraste, fluxo de contrapressão e de atrito entre as partículas ${ }^{[7]}$. Dessa forma, durante a mistura, a fase dispersa sofre um processo de cominuição (quebra das gotas) progressiva até atingir um diâmetro mínimo da gota. Com a diminuição do diâmetro, a quebra tornase cada vez mais difícil. Alguns trabalho ${ }^{[5]}$ mostraram que o tamanho final da partícula da fase dispersa, no entanto, é maior que o previsto, e esse diâmetro aumenta com o teor da fase dispersa. Uma justificativa para tal fenômeno é o efeito da coalescência, gerado pela colisão das gotas da fase dispersa durante a mistura no estado fundido.

Tokita ${ }^{[5]}$ sugeriu que o diâmetro final de equilíbrio da gota na mistura é definido pela relação entre as taxas de cominuição e de coalescência das gotas da fase dispersa. Uma maneira de se calcular as taxas de quebra das partículas $\left(K_{\text {cominuição }}\right)$ e de coalescência $\left(K_{\text {coalescência }}\right)$ é através das seguintes equações:

$$
\begin{aligned}
& K_{\text {cominuisão }}=\frac{\eta_{d} \dot{\gamma}^{2}}{E_{D K}+6 \frac{v}{d}} \\
& K_{\text {coalescência }}=\frac{4}{\pi} P_{r} \phi_{d} \dot{\gamma}
\end{aligned}
$$

Onde $\eta_{d}$ é a viscosidade da fase dispersa, $\dot{\gamma}$ é a taxa de cisalhamento, $E_{D K}$ é a energia para romper a partícula ou gota, $v$ é a tensão interfacial, $d$ é diâmetro da partícula, $P_{r}$ é a probabilidade de colisão e $\phi_{d}$ é a fração volumétrica da fase dispersa.

$\mathrm{Na}$ condição de equilíbrio, ou seja, $K_{\text {cominuição }}=K_{\text {coalescência }}$, obtém-se o diâmetro de equilíbrio da partícula $\left(d_{e q}\right)$ :

$$
d_{e q}=\frac{24 P_{r} v}{\pi}\left(\frac{\phi_{d}}{\eta_{d} \dot{\gamma}-E_{D K} \frac{4}{\pi} P_{r} \phi_{d}}\right)
$$

Essa equação mostra que o diâmetro de equilíbrio da gota aumenta com a concentração e com o coeficiente de tensão interfacial ( $v)$ e diminui com a tensão de cisalhamento $(\tau=\eta \gamma)$.

\section{Blendas PVC/NBR}

Uma das mais importantes blendas poliméricas miscíveis comerciais é PVC/NBR ${ }^{[8]}$, desenvolvida em 1942, sendo a primeira blenda termoplástica comercial, onde a borracha nitrílica era tida como plastificante polimérico permanente do $\mathrm{PVC}^{[1]}$.

As borrachas nitrílicas são os principais modificadores poliméricos em compostos de PVC na forma de blendas poliméricas, e podem ser consideradas plastificantes poliméricos de alto peso molecular, alta permanência, porém com baixa eficiência de plastificação ${ }^{[9]}$. A borracha NBR promove melhoria na resistência ao ozônio, envelhecimento e resistência química do PVC, enquanto que o PVC melhora as propriedades de abrasão, rasgamento e tração e as propriedades do extrudado da blenda da NBR ${ }^{[8]}$.

\section{Poli (cloreto de vinila) - PVC}

O poli (cloreto de vinila) ocupa um lugar de destaque entre os termoplásticos presentes no cotidiano. O PVC, devido à necessidade de incorporação de aditivos durante o seu processamento, apresenta características que o tornam adaptável a múltiplas aplicações, sendo considerado um dos plásticos mais versáteis ${ }^{[9]}$.

Aproximadamente $80 \%$ do PVC consumido no mundo é produzido através de polimerização do monômero cloreto de vinila (MVC) em suspensão. Por este processo é possível obter resinas de PVC adequadas à produção de tubos e conexões, perfis, isolamento de fios e cabos elétricos, entre outras aplicações $^{[9]}$.

A presença do átomo de cloro na estrutura química do PVC o torna naturalmente resistente à propagação de chamas. Além disso, confere polaridade, que permite sua mistura com uma gama de aditivos muito maior que a de qualquer outro termoplástico, possibilitando a preparação de formulações com propriedades e características adequadas a cada aplicaçã $0^{[9]}$. As resinas de PVC obtidas pelo processo de

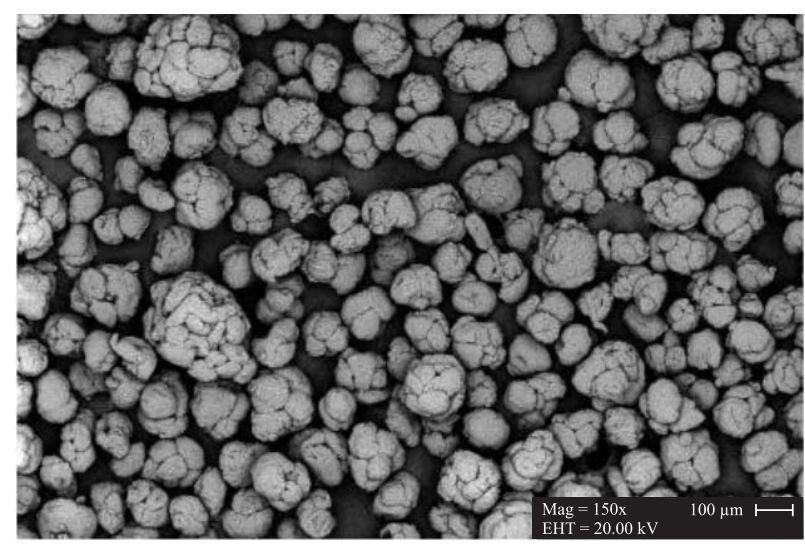

Figura 4. Microscopia eletrônica de varredura de uma resina de PVC obtida pelo processo de polimerização em suspensão ${ }^{[9]}$ 
polimerização em suspensão consistem em partículas com diâmetros na faixa de 50 a $200 \mu \mathrm{m}$. A Figura 4 apresenta uma micrografia da resina de PVC obtida pelo processo de suspensão $0^{[9]}$.

O PVC é considerado um polímero amorfo ou de baixa cristalinidade (máxima de 15\%), devido à disposição espacial randômica dos átomos de cloro na cadeia polimérica. $\mathrm{O}$ PVC é susceptível a reações de degradação quando exposto ao calor, a agentes oxidantes e ao intemperismo, afetando suas propriedades durante o processamento e uso final. A aditivação do PVC proporciona a obtenção de compostos que são possíveis de serem processados, possibilitando a obtenção de uma ampla gama de produtos com as características desejáveis para cada aplicação ${ }^{[9]}$.

\section{Borracha nitrílica - NBR}

A borracha nitrílica é um elastômero sintético e, de uma maneira geral, pode ser definida como sendo um copolímero de um dieno e de uma nitrila insaturada. Esses elastômeros são obtidos através de copolimerização em emulsão de acrilonitrila e butadieno, formando copolímeros elastoméricos estatísticos acrilonitrila-butadieno (NBR). O processo de emulsão pode ser realizado a quente ou a frio. As borrachas polimerizadas a quente distinguem-se por melhores propriedades de adesão, flexibilidade e deformação permanente por compressão. Em contraposição, as borrachas obtidas em emulsão a frio apresentam melhores características físicas e são mais facilmente processáveis ${ }^{[10]}$.

A acrilonitrila, presente na estrutura da NBR, é um monômero vinílico e é composta por dois carbonos onde o grupo ciano $(\mathrm{C} \equiv \mathrm{N})$ substitui um átomo de hidrogênio. O grupo ciano é altamente polar e responsável pela elevada resistência a óleos desses elastômeros. As massas molares dos monômeros de butadieno e acrilonitrila são muito próximas, fazendo com que a proporção em massa seja praticamente igual à proporção em número de moléculas para este elastômero ${ }^{[10]}$.

Os tipos ou grades de NBR são determinados pelo teor de acrilonitrila presente na sua composição. De maneira geral, quanto maior o teor de acrilonitrila, maior a resistência mecânica e a resistência à óleos e solventes, porém menor a elasticidade e a flexibilidade ${ }^{[10]}$.

\section{Estado de Mistura de blendas PVC/NBR}

$\mathrm{O}$ estado de mistura de sistemas PVC/NBR está diretamente relacionada ao teor de acrilonitrila da borracha nitrílica, conforme já mencionado anteriormente. Liu e colaboradores $^{[11,12]}$ estudaram a influência da adesão interfacial na tenacidade sob impacto de blendas PVC/NBR (50/50). Observaram que aumentando o teor de acrilonitrila na NBR de 18 para $26 \%$ (em massa), a adesão interfacial entre PVC e NBR aumentou e o tamanho de partículas de borracha na matriz diminuiu. Esses estudos mostraram que a interação molecular entre PVC e NBR aumenta com o aumento do teor de acrilonitrila do NBR, melhorando, conseqüentemente, a adesão interfacial e por conseqüência obtiveram um aumento significativo na tenacidade.
Wang e colaboradores ${ }^{[13]}$, por sua vez, estudaram a utilização de pós-ultrafinos de borracha vulcanizada (UFPR) para desenvolver composto de PVC rígido com elevada tenacidade e resistência ao calor. O teor de NBR utilizado foi de 7 $\%$ (em massa). Os estudos mostraram que PVC e NBR são compatíveis e a compatibilidade aumenta com o teor de acrilonitrila da NBR, sendo que os resultados foram otimizados para teor de $33 \%$ de acrilonitrila. Com esse teor, observa-se o aumento do grau de miscibilidade da blenda $\mathrm{PVC} / \mathrm{NBR}$, o que influencia nas propriedades de resistência à tração e ao impacto, notadamente pelo aumento da adesão interfacial entre as fases.

O PVC e a NBR são polímeros polares e podem ser miscíveis. Verifica-se que o grau de miscibilidade aumenta com o teor de acrilonitrila no NBR, como conseqüência da polaridade, até um teor ótimo a partir da qual se observa o comprometimento das propriedades mecânicas, gerando blendas com morfologia de fases aglomeradas e heterogêneas. Dessa forma, o estudo da dispersão da fase borrachosa na matriz de PVC é muito importante para compreender as propriedades finais da blenda, como, por exemplo, o efeito da tenacidade através da atuação do mecanismo de deformação da blenda quando submetida ao ensaio de impacto.

\section{Dispersão da fase borrachosa em blendas PVC/NBR}

A incorporação da borracha na matriz de PVC foi estudada por Kwak e Nakajima ${ }^{[14]}$, que monitoraram o processo de mistura e homogeneização, em reômetro de torque, de NBR em uma blenda NBR/PVC, utilizando-se de microscopia
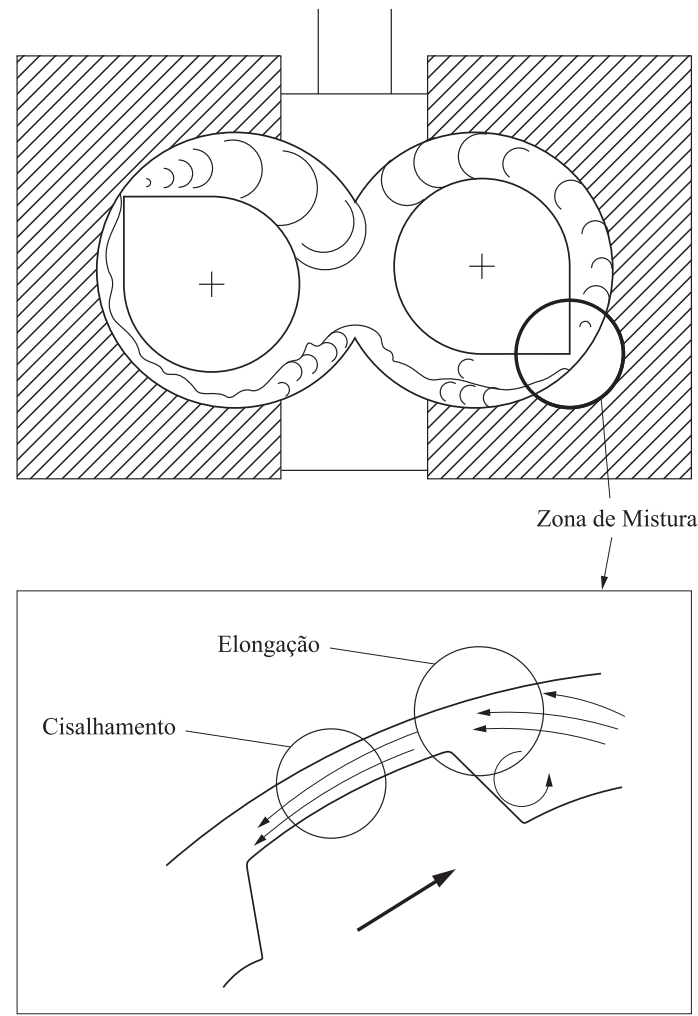

Figura 5. Esquema da deformação e do comportamento do material no interior do misturador 


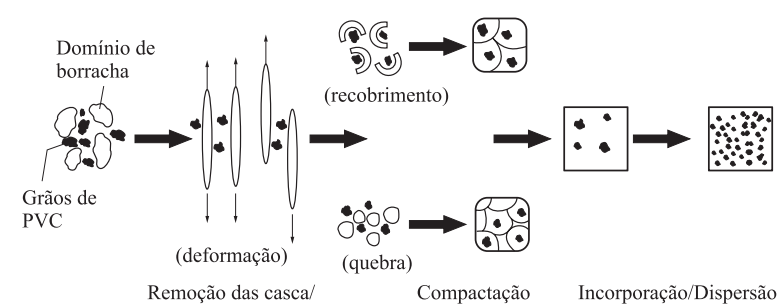

Compactação Incorporação/Dispersão

Figura 6. Modelo proposto por Kwak e Nakajima ${ }^{[14]}$ para o mecanismo de homogeneização, envolvendo remoção de cascas e quebra do PVC

eletrônica de varredura (MEV), para determinados tempos de mistura. Um esquema do misturador é apresentado na Figura 5. Há duas características geométricas no interior do misturador: (1) a zona de mistura entre as lâminas do rotor e as paredes da câmara e (2) a distância entre os dois rotores.

Na zona 1 (ou zona de mistura) o material se movimenta nas direções axial e lateral, resultando numa quebra longitudinal e sobreposição lateral. Uma mistura efetiva ocorre na zona de moagem, onde a tensão deformacional é grande. Nesta zona o material é deformado em pequena e grande escala (deformação elongacional). Quando partes de NBR passam através da zona de moagem, estas sofrem grande deformação por cisalhamento, com elevada tensão, atuando como transferidor de tensão dos rotores para as partículas de PVC. Este processo quebra as partículas de PVC que serão incorporadas na borracha. A partir do monitoramento por microscopia eletrônica de varredura das misturas da blenda NBR/PVC, Kwak e Nakajima ${ }^{[14]}$ elaboraram um modelo para descrever o mecanismo de homogeneização dessas blendas, apresentado na Figura 6.

Liu e colaboradores ${ }^{[15-18]}$ publicaram uma série de artigos que correlacionam os parâmetros morfológicos de blendas poliméricas binárias, principalmente do sistema PVC/NBR. Segundo $\mathrm{Liu}^{[15]}$, no caso da blenda PVC/NBR, esta deverá apresentar, no mínimo, duas fases distintas: a fase matriz e a fase dispersa. Geralmente, as partículas da fase dispersa estão distribuídas de forma randômica ou ao acaso na matriz. Para a maioria dos sistemas binários observam-se três morfologias de dispersão de fase borrachosa, esquematizadas na Figura 7.

Na morfologia de fases bem dispersas, as partículas de borracha estão aleatoriamente dispersas na matriz termoplástica. A morfologia "pseudo-network" é composta por partículas de borracha dispersas na matriz termoplástica, formando uma pseudo-rede e a morfologia de partículas aglomeradas é formada por aglomerados de borracha espalhados na matriz termoplástica ${ }^{[15]}$.

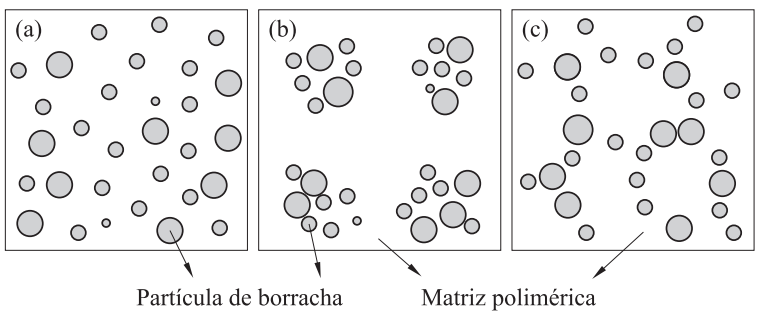

Figura 7. Esquema da distribuição espacial das partículas de borracha: (a) bem dispersa; (b) aglomerada; (c) "pseudo-network"
A otimização de propriedades é esperada para blendas que possuam morfologia com fase borrachosa bem dispersa. Para a maioria dos sistemas poliméricos bifásicos, a distribuição de tamanho de partículas da fase dispersa é dada por uma distribuição estatística normal. Para sistemas polidispersos, a conFiguração das partículas na matriz polimérica, ou seja, a maneira como estão dispersas nesta matriz, pode ser expressa pela seguinte equação, desenvolvida por Liu:

$$
T(d, \sigma, \phi)=d\left[\left(\frac{\pi}{6 \phi}\right)^{\frac{1}{3}} \exp \left(1,5 \ln ^{2} \sigma\right)-\exp \left(0,5 \ln ^{2} \sigma\right)\right]
$$

Onde $T$ é a configuração das partículas da matriz, $d$ é o tamanho das partículas, $\phi$ é a fração volumétrica de partículas e $\sigma$ representa uma relação da distribuição do tamanho das partículas. Os parâmetros $d, \phi$ e $\sigma$ são obtidos experimentalmente. De acordo com a proposta de $\mathrm{Liu}^{[15]}$, a equação desenvolvida pode ser utilizada para o sistema PVC/NBR. A equação prediz que a espessura de ligamento médio da matriz $(T)$ aumenta com o tamanho e distribuição de tamanho das partículas e com a diminuição da fração volumétrica das partículas, sendo que o efeito da distribuição do tamanho de partículas é um fator determinante para a tenacificação desses sistemas, por exemplo.

Segundo $\mathrm{Liu}^{[16]}$, para blendas $\mathrm{PVC} /$ borracha existem três estados de dispersão da fase borrachosa. A Figura 8 apresenta um esquema da dispersão da fase borrachosa na matriz de PVC.
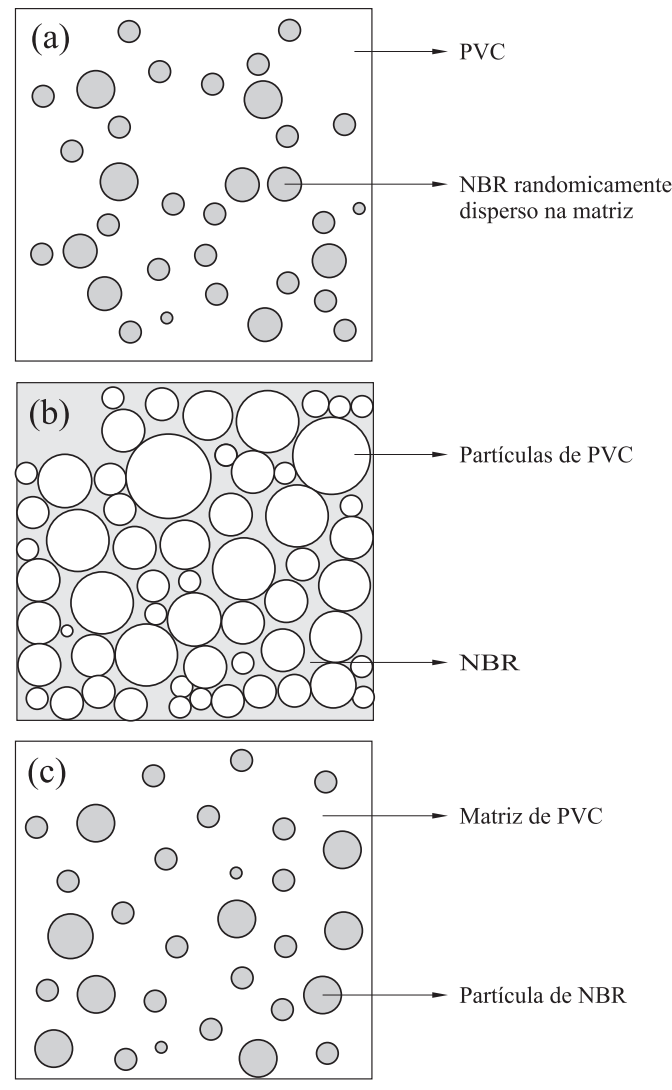

Figura 8. Esquema da dispersão da fase borrachosa em blendas PVC/borracha: (a) "pseudo-network"; (b) "network"; (c) partículas bem dispersas 


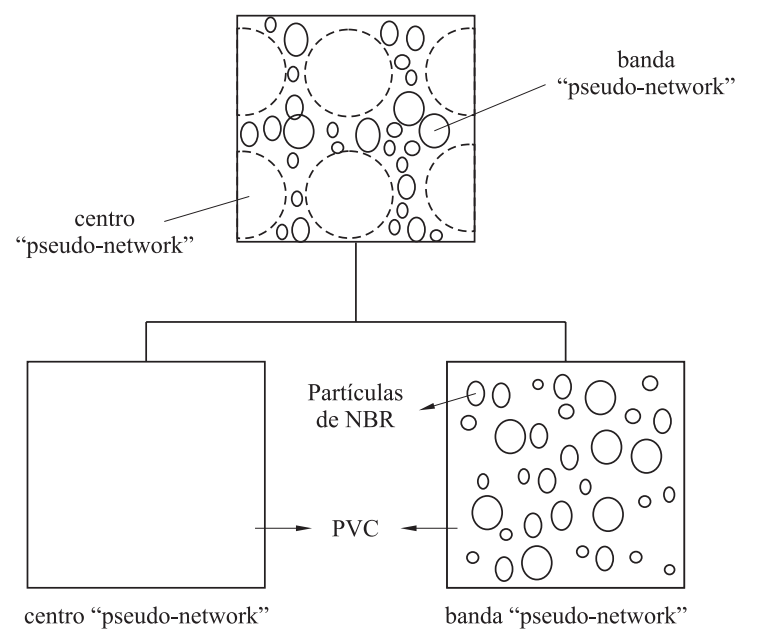

Figura 9. Esquema da morfologia "pseudo-network"

A morfologia "pseudo-network" é caracterizada por partículas de borracha randomicamente dispersas entre as partículas primárias de PVC, tendo como exemplos deste estado as blendas PVC/ABS e PVC/MBS. Na morfologia "network" há duas fases contínuas e as partículas primárias de PVC estão principalmente na forma de estrutura particulada. Essa morfologia é típica de sistemas PVC/NBR. Uma morfologia com partículas bem dispersas é a mais desejada em blendas poliméricas uma vez que proporcionam aumento efetivo de algumas propriedades, tais como resistência à tração e tenacidade sob impacto ${ }^{[16]}$.

De acordo com Liu ${ }^{[18]}$, a morfologia "pseudo-network" apresentada na Figura 9 é composta por duas fases: (1) bandas "pseudo-network", que contém partículas de borracha uniformemente dispersas na banda e PVC fundido, e (2) centro "pseudo-network" que consiste de partículas primárias de PVC.

Em morfologia com partículas bem dispersas o aumento da fração volumétrica da borracha gera aumento no tamanho médio das partículas de borracha. Tal fator é crítico uma vez que as propriedades das blendas poliméricas são amplamente afetadas pelo tamanho e distribuição de tamanho das partículas da fase dispersa ${ }^{[16]}$.

\section{Comentários Finais}

Os termoplásticos modificados com elastômeros constituem uma classe de materiais que podem apresentar uma ótima relação custo/benefício. Uma grande vantagem consiste em utilizar, para a obtenção da blenda, os mesmos equipamentos para o processamento de termoplásticos e obter produtos com características particulares, com maior valor agregado.

A fase borrachosa deve ser dispersa em pequenas partículas na matriz termoplástica com tamanho de partículas adequado para proporcionar as modificações desejadas na matriz polimérica. A definição do tamanho e da distribuição de tamanho das partículas da fase dispersa em uma matriz polimérica depende de vários fatores entre os quais a interação entre as fases, as características do equipamento e as condições de mistura utilizadas. Se há uma boa interação entre os componentes da blenda, as partículas de borracha dispersas na matriz termoplástica poderão ser pequenas o suficiente para atuar na tenacificação da blenda e na melhoria das propriedades de impacto e tração; se não ocorre interação favorável entre a fase dispersa e a matriz, a borracha pode se apresentar como partículas de dimensão elevada e não adequadas para promover a tenacificação da blenda.

No caso especifico do sistema PVC e NBR, estudos comprovaram que a miscibilidade/interação entre as fases e a dispersão da fase borrachosa em blendas PVC/NBR estão intimamente relacionadas com o desempenho final desta blenda polimérica. Estudos de Liu e seus colaboradores ${ }^{[11,12,15-18]}$ mostraram que o grau de miscibilidade das fases PVC e NBR aumenta com a porcentagem de acrilonitrila da borracha nitrílica, havendo um teor ótimo na qual há melhora nas propriedades mecânicas. A morfologia "pseudo-network" promoveu melhoras quantitativas na resistência ao impacto quando comparada à morfologia de partículas bem dispersa.

A dispersão da fase borrachosa depende de muitos fatores como concentração da fase borrachosa, tamanho e distribuição de tamanho das partículas e fração volumétrica da fase dispersa. De maneira geral, aumentando-se a concentração da fase borrachosa, observa-se uma diminuição do módulo elástico e da resistência à tração. Dessa forma, dependendo da aplicação da blenda PVC/NBR, deve-se relacionar tais fatores para que se obtenha a otimização das propriedades.

\section{Referências Bibliográficas}

1. Utracki, L. A. - "Polymer alloys and blends: thermodynamics and rheology", Hanser Publishers, New York (1989).

2. Hage, E.; Pessan, L. A. - "Aperfeiçoamento em tecnologia de plásticos. Módulo 7: blendas poliméricas", ABPol, São Carlos (2001).

3. Paul, D. R.; Newman, S. - "Polymers blends", vol.1, Academic Press, New York (1978).

4. Paul, D. R.; Barlow, J. W.; Keskkula, H. - "Encyclopedia of polymer science and engineering", vol.12, 2a ed., John Wiley \& Sons, New York (1988).

5. Utracki, L. A.; Shi, Z. H. - Pol. Eng. Sci., 32, p.1824 (1992).

6. Han, C. D. - "Multiphase flow in polymer processing", Academic Press, New York (1981).

7. Tadmor, Z.; Gogos, C. - "Principles of polymer processing", John Wiley \& Sons, New York (1979).

8. George, K. E.; Rani, J.; Francis, D. J. - J. Appl. Pol. Sci., 32, p. 2867 (1986).

9. Rodolfo Jr., A.; Nunes, L. R.; Ormanji, W. - "Tecnologia do PVC", 2a . ed., Proeditores, São Paulo (2006). 
10. Morrill, J. P - "Rubber technology", Krieger Publishing Company, Melbourne (1981).

11. Liu, Z. H.; Zhu, X.; Wu, L.; Li, Y.; Qi, Z.; Choy, C.; Wang, F. - Polymer, 42, p. 737 (2001).

12. Liu, Z. H.; Wu, L.X.; Kwok, K. W.; Zhu, X. G.; Qi, Z. N.; Choy, C. L.; Wang, F. S. - Polymer, 42, p. 1719 (2001).

13. Wang, Q.; Zhang. X.; Liu, S.; Gui, H.; Lai, J.; Liu, Y.; Gao, J.; Huang, F.; Song, Z.; Tan, B. H.; Qiao, J. Polymer, 46, p. 10614 (2005).

14. Kwak, S. Y.; Nakajima, N. - Macromolecules, 29, p. 5446 (1996).
15. Liu, Z. H.; Zhang, X. D.; Zhu, X. Z.; Li, R. K. Y.; Qi, Z. N.; Wang, F. S.; Choy, C. L. - Polymer, 38, p. 5267 (1997).

16. Liu, Z. H.; Zhang, X. D.; Zhu, X. Z.; Li, R. K. Y.; Qi, Z. N.; Wang, F. S.; Choy, C. L. - Polymer, 39, p. 5019 (1998).

17. Liu, Z. H.; Zhang, X. D.; Zhu, X. Z.; Qi, Z. N.; Wang, F. S.; Li, R. K. Y.; Choy, C. L. - Polymer, 39, p. 5027 (1998).

18. Liu, Z. H.; Zhu, X. Z.; Li, R. K. Y.; Qi, Z. N.; Wang, F. S. - Polymer, 39, p. 5035 (1998). 